\title{
Study of light-induced structural changes associated with Staebler-Wronski Photo-degradation in micro-crystalline silicon thin films
}

\author{
Sucheta Juneja, S. Sudhakar, Kalpana Lodhi, Srishti Chugh, Mansi Sharma and Sushil Kumar \\ CSIR Network of Institutes for Solar Energy, CSIR - National Physical Laboratory, \\ Dr. K.S. Krishnan Marg, New Delhi, India 110012 \\ E-mail: skumar@nplindia.org
}

\begin{abstract}
Hydrogenated amorphous silicon based devices lacks behind in their fruitful applications as it suffers from major drawback i.e. light induced degradation or S-W effect. Various theories or literature have been discussed so far, but exact mechanism is still an open challenge in research community. We report on light-induced structural changes in amorphous and micro/nano crystalline silicon by performing light soaking experiments for nearly $8 \mathrm{hrs}$. Under vacuum accompanied with the effect of annealing on these films. The electrical, structural and optical properties were analyzed with the use of dark and photo conductivity measurements, Raman spectroscopy, Scanning electron microscopy (SEM) and Photoluminescence studies. Using Raman spectroscopy, we estimated the bond distortion w.r.t degradation percentage in samples. We observed that crystallinity as well as particle size are responsible for increase or decrease in degradation of photoconductivity. Having $\mathbf{7 2 . 2 5 \%}$ crystallinity highly stable microcrystalline silicon films showed $1.44 \%$ photo-degradation.
\end{abstract}

Index Terms - amorphous \& microcrystalline silicon, Staebler - Wronski effect, Light-Induced degradation.

\section{INTRODUCTION}

The research on hydrogenated amorphous silicon (a-Si:H) devices was started in 1976 by Carlson and Wronski with the development of PV devices [1]. Photo-induced degradation in amorphous silicon (a-Si) materials is a major problem which should be solved for fabrication of efficient \& stable solar cells [2]. The origin of large structural changes in a-Si:H after light exposure is still unclear. Efforts have been made to understand and overcome this problem but not yet fully understood, there is no consciences among researchers on single mechanism responsible for explaining the nature of light induced defects in amorphous silicon [3, 4]. It was found that degradation was caused due to increased danglingbond defect density upon light soaking which reduces the lifetime of photo-excited free carriers with enhanced recombination through the electronic localized states formed by the presence of dangling bonds in the network, leading to deterioration of efficiency in a-Si:H based solar cells [5]. The dangling bond in amorphous silicon contributes to defects which arise due to distortion from the well ordered $\mathrm{sp}^{3}$ symmetry of crystalline silicon. These defects lead to undesirable electrical properties, resulting in electrons recombination center rather than contributing electric current. The drawback of a-Si:H can overcome upto certain extent by the use of micro/nano-crystalline silicon ( $\mu \mathrm{c} / \mathrm{nc}-\mathrm{Si}: \mathrm{H})$.
Recently, microcrystalline silicon has attracted great interest for their potential applications most importantly that such solar cells do not suffer from the notorious light-induced degradation. The most important features of microcrystalline film are its microstructures, e.g. the distribution of amorphous and crystalline phase or disordered regions including grain boundaries and voids within the film. The microcrystalline silicon has several nanometer-sized grains dispersed within amorphous silicon plus grain boundaries which increases the absorption of light and electron mobility results in good resistance to light-induced degradation under prolonged light illumination. Thus, mixed micro/nano crystallites embedded in amorphous matrix could be a solar material with high stability to light soaking over conventional amorphous films $[6,7]$. It is understandable that on light soaking the amorphous \& crystalline volume fractions and grain boundary phases, attributed to the light-induced degradation in micro crystalline silicon films. Thus, it is important to understand light induced degradation in mixed - phase microcrystalline silicon films, particularly as a function of crystalline volume fraction and crystallites/particle size, so that arrest/minimize of photo-degradation in this material can be possible.

\section{EXPERIMENTAL DETAILS}

Intrinsic mixed phase of a-Si:H and $\mu \mathrm{c} / \mathrm{nc}-\mathrm{Si}: \mathrm{H}$ thin films were deposited on corning 7059 and TCO coated glass substrates using capacitively coupled plasma enhanced chemical vapor deposition (PECVD) technique at $60 \mathrm{MHz}$ assisted VHF (very high frequency) power. Films were grown using $\mathrm{SiH}_{4}$ and $\mathrm{Ar}$ gaseous mixture at $200{ }^{\circ} \mathrm{C}$ substrate temperature while maintaining the chamber pressure at 0.25 Torr and power $20 \mathrm{~W}$. The effect of variation of argon dilution was investigated systematically on deposition of $\mu \mathrm{c} / \mathrm{nc}-\mathrm{Si}: \mathrm{H}$ films for obtaining various crystallinity in these films . Raman spectroscopy (LabRAM HR800 JY) with a He- Ne (633 nm) laser was used for the determination crystalline volume faction, particle size and angular distortion in the structural network. The particle size was also estimated using PL spectra. The thickness of deposited films was determined by a stylus based thickness profiler (Ambios XP 200, USA). Lightsoaking measurement was performed for $8 \mathrm{hrs}$. By monitoring photoconductivity (Keithley 617 programmable electrometer) of $\mu \mathrm{c} / \mathrm{nc}-\mathrm{Si}: \mathrm{H}$ films which were kept in vacuum $\left(10^{-5}\right.$ Torr) using $100 \mathrm{~mW} / \mathrm{cm}^{2}$ illumination. A further check on the 
crystalline growth structure of such deposited $\mu \mathrm{c}-\mathrm{Si}: \mathrm{H}$ films were investigated by Scanning electron microscopy.

\section{RESULTS \& DISCUSSIONS}

Raman spectroscopy is an efficient and sensitive technique in determination of small changes in crystallinity. Using Raman spectra, we also calculated distortions in structural network. Fig. 1 and Fig. 2 show the Raman spectra of deposited films before and after light-soaking. It is clear from figures that before Light soaking all films are microcrystalline in nature but after Light soaking shows that the microstructures in biphasic material changes to amorphous. Information about the crystalline volume fraction of the material is useful to scrutinize the stability of films [8] and information regarding size of nanocrystals is important to understand the role of quantum confinement of electrons [9]. These results indicate that the crystalline phase reduced and amorphous \& grain boundary component increased after light soaking consistently. Interestingly after light soaking crystallite size of these films also reduced. Thus, there may be correlation exist between crystallite size and crystalline fractions with light induced photo-degradation, which needs to be investigated.

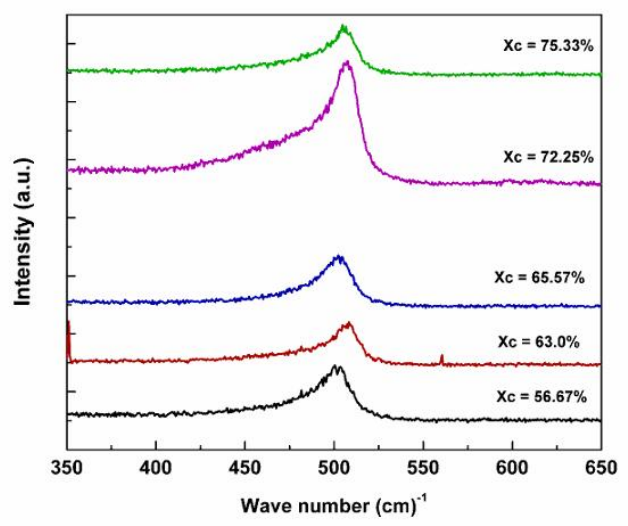

FIG.1. Raman spectra of $\mu \mathrm{c} / \mathrm{nc}-\mathrm{Si}: \mathrm{H}$ films before Light soaking.

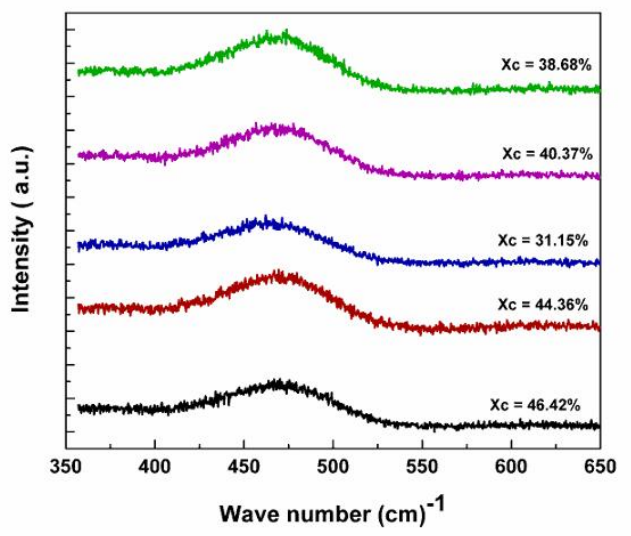

FIG. 2. Raman spectra of $\mu \mathrm{c} / \mathrm{nc}-\mathrm{Si}: H$ films after Light soaking .

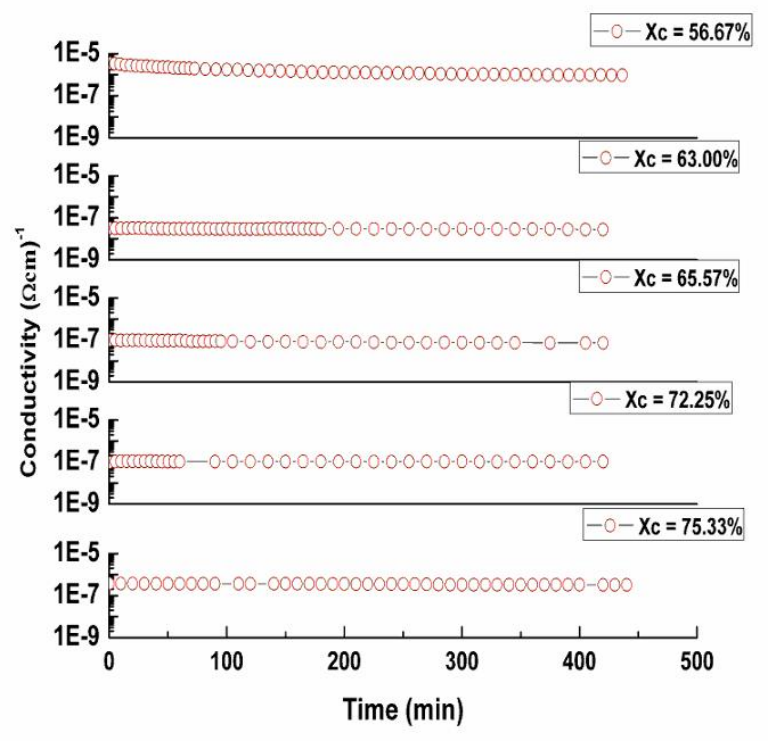

FIG.3. Light soaking of samples at different crystalline fraction.

From Fig. 3 it can be seen that there is an optimum crystalline fraction in the range $65 \%$ to $75 \%$ which showed lowest photodegradation (less than 20\%). These films also contain large variation in particle size. It is well known that amorphous silicon degrade due to breaking of strained $\mathrm{Si}-\mathrm{H}$ and $\mathrm{Si}-\mathrm{Si}$ bonds. The significant amount of photo-degradation observed in these microcrystalline films may be due to large strained network. Thus, lattice distortions (distortion in bond angle) of these films were also estimated using Raman spectra before and after light-soaking and the variation of lattice distortion as a function of crystalline volume fraction.

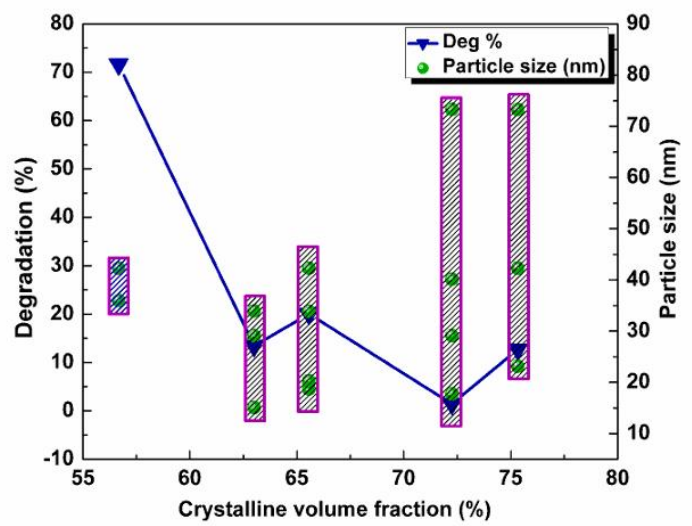

FIG.4. Variation in particle size and degradation percentage w.r.t crystalline volume fraction.

It is observed that as crystalline fraction increases degradation percentage decreases. These films also contain large variation in particle size. Non-uniform distribution of particle size may leads to more stress and that may be responsible for photodegradation. So, we can say that photo-degradation not only depends on bonded/unbounded hydrogen content and 
crystalline fraction but also on particle size and defect density in the material. It is clear from figure 4 that the crystalline films having crystallinity above $70 \%$ have large variation in crystallite sizes \& also larger size crystallites and also found to have less degradation in these films. It is well known that large size crystallite contain less strain which may also be reason for lower photo degradation. Whereas, the film having small size crystallites suffers higher degradation this may be due to the reason that the small size particles absorb weakly the higher order of wavelength (as in SEM micrograph, fig. 6 having Xc 56.67\%) in the solar spectrum. This we may be understand as if photon energy is larger than band gap energy, some energy is wasted and electrons quickly thermalize back down to conduction band edges and this thermalize energy further add to strain in the network. Whereas, absorption probability of large size particles (as in SEM micrograph, fig. 6 having Xc $75.33 \%$ consisting of various size of particles) is more which may result in less degradation. For Photovoltaic applications, sufficient amount of energy is needed to efficiently absorb to create an electron-hole pair. From the data obtained, we find that materials with very high crystallinity may result low degradation.
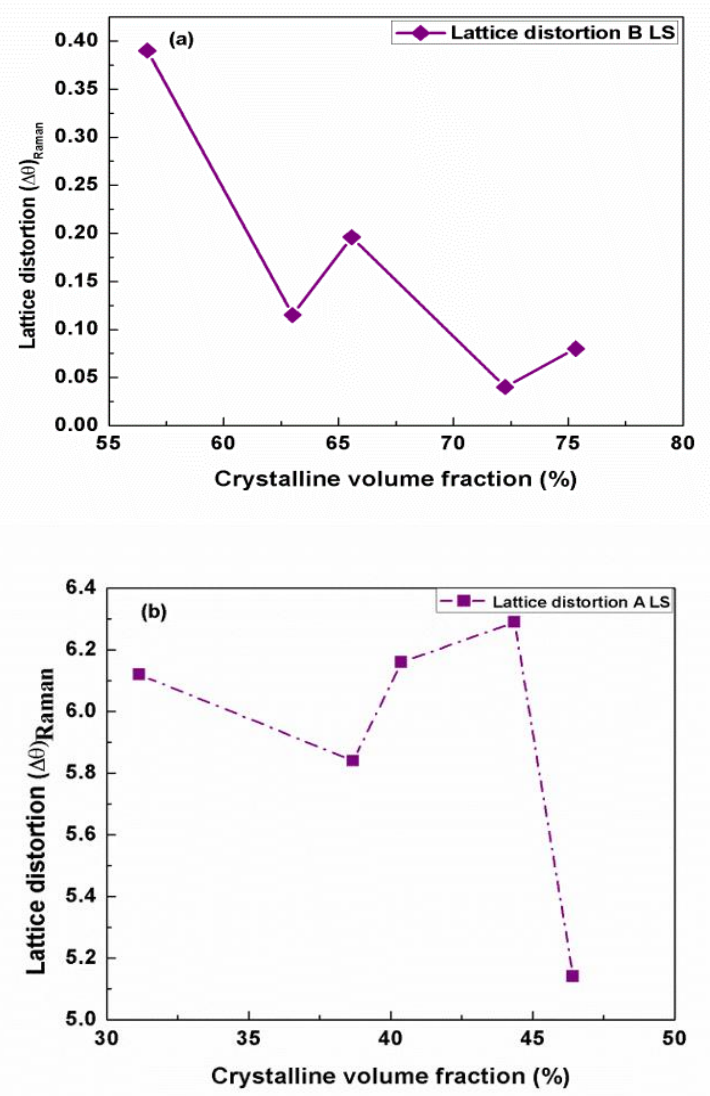

Fig. 5. Lattice distortion estimated from Raman Spectroscopy (a) before light -soaking and (b) after light-soaking.

We have also calculated lattice distortion in these films using Raman spectra. The estimated change in lattice distortion before and after light soaking w.r.t crystalline volume fraction were found to correlate well with photo degradation as shown in figures $4 \& 5$. It is clear that as crystallinity in the film increases, the angular distortion i.e. deviation in bond angle decreases whereas there is large change in angular distortion observed after light soaking. This value range varies from 0.04-0.4 to 5.1-6.3 with the change in crystalline volume fraction from Xc: $56-75$ (before light soaking) to Xc: $30-45$ (after light soaking). It is clear from the results that lattice distortion increases the stress/strain in the network which may leads to increase in disorder or randomness in structure and cause for photo-degradation in $\mu \mathrm{c}-\mathrm{Si}: \mathrm{H}$ films. These results found to correlate well with light induced degradation of photoconductivity. So, we can conclude that there are large structural changes arises in the structure after light soaking.

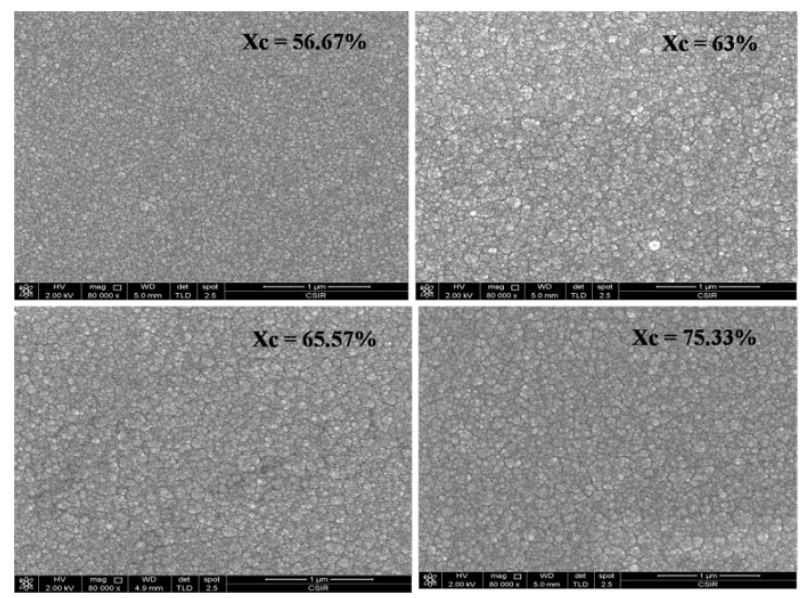

Fig. 6. SEM images of $\mu \mathrm{c} / \mathrm{nc}-\mathrm{Si}: \mathrm{H}$ films having different crystalline volume fraction.

Fig.6. shows distribution of crystallites within a-Si:H matrices for various $\mu \mathrm{c} / \mathrm{nc}-\mathrm{Si}: \mathrm{H}$ films. It is clearly revealed from this figure that crystallite size is smaller in the film having lower crystalline volume fraction $(56.67 \%)$ whereas film having larger crystalline volume fraction $(75.33 \%)$ showed bigger crystallite. Usually bigger crystallites film contains lower strain and which may be reason for lower photo-degradation observed in the present investigation. In addition it is also clearly observed from these SEM micrographs that the size and distribution of crystallites is non-uniform leads to mismatch in the bonding network or distortion which may result in photo-degradation.

\section{CONCLUSIONS}

The structural heterogeneity associated with $\mu \mathrm{c}-\mathrm{Si}: \mathrm{H}$ were shown using Raman spectroscopy. It was found that photodegradation in these films decreases with the increase of crystallinity. It seems that photo-degradation also depends on crystallite size which needs to be investigated further. Lattice distortion i.e. strain in the network of $\mu \mathrm{c}-\mathrm{Si}: \mathrm{H}$ films were estimated using Raman spectroscopy and found to be correlated well with photo-degradation. Microcrystalline film having $72.25 \%$ crystallinity showed only $1.44 \%$ of photodegradation and fabrication of solar cells using these films is in progress. 


\section{ACKNOWLEDGEMENTS}

The authors are thankful to Director, CSIR-NPL, and New Delhi for his kind support and encouragement. We are also thankful to CSIR-India (for TAPSUN program) and MNRE, Govt. of India for the financial support (Sanction No. 31/29/2010-11/PVSE).

\section{REFERENCES}

[1] D.E.Carlson, C.R.Wronski, Appl. Phys. Lett., 28, 671

(1976).

[2] D.L. Staebler, C.R. Wronski, Appl. Phys. Lett. 31 (1977) 292.

[3] H. Fritzsche, Annual Rev Mater Res 31, 47(2001).

[4] H. Fritzsche, Mat. Res. Soc. Proc.1245, A14.01 (2010).

[5] M. Stutzmann, W.B. Jackson, C.C. Tsai, Phys. Rev. B 32 (1985) 23.

[6] J. Meier, R. Fluckiger, H. Keppner, and A. Shah, Appl. Phys. Lett. 65,860 (1994).

[7] D. V. Tsu, B. S. Chao, S. R. Ovshinsky, S. Guha, and J. Yang, Appl. Phys.Lett. 71, 1317 (1997).

[8] Myong, S. Y.; Sriprapha, K.; Yashiki, Y.; Miyajima, S.; Yamada, A.; Konagai, M. Solar Energy Mater. Solar Cells 2008, 639, 92.

[9] Droz, C.; Vallat-Sauvain, E.; Bailat, J.; Feitknecht, L.; Meier, J.;Shah, A. Solar Energy Mater. Solar Cells 2004, 81, 61. 